\title{
Rhodium-Catalyzed Asymmetric 1,4-Addition of Arylboronic Acids to Coumarins: Asymmetric Synthesis of (R)-Tolterodine
}

\author{
Gang Chen, Norihito Tokunaga and Tamio Hayashi* \\ Department of Chemistry, Graduate School of Science, Kyoto University, \\ Sakyo, Kyoto 606-8502, Japan
}

\section{Supporting Information}

\section{General}

All manipulations were carried out with standard Schlenk techniques under nitrogen. NMR spectra were recorded on a JEOL JNM LA-500 spectrometer (500 $\mathrm{MHz}$ for ${ }^{1} \mathrm{H}$, and $125 \mathrm{MHz}$ for ${ }^{13} \mathrm{C}$ ). Chemical shifts are reported in $\delta \mathrm{ppm}$ referenced to an internal $\mathrm{SiMe}_{4}$ standard for ${ }^{1} \mathrm{H}$ NMR and chloroform- $d(\delta 77.05)$ for ${ }^{13} \mathrm{C} \mathrm{NMR}$. Optical rotations were measured on a JASCO DIP-370 polarimeter.

1,4-Dioxane, THF and toluene were distilled over benzophenone ketyl under nitrogen. Diisopropylamine was distilled from $\mathrm{CaH}_{2}$ under nitrogen prior to use. 6Methylcoumarin (TCI), coumarin (Nacalai Tesque), 7-methoxycoumarin (Acros) and phenylboronic acid (TCI) were used as received. (R)-Segphos, ${ }^{1}(R)$-P-Phos, ${ }^{2}(R)$ Binap, ${ }^{3} \mathrm{Rh}(\mathrm{acac})\left(\mathrm{C}_{2} \mathrm{H}_{4}\right)_{2},{ }^{4}$ 6-methoxycarbonylcoumarin, ${ }^{5}$ methyl $(E)$-3-(2-methoxy-5methylphenyl)propenoate, ${ }^{6} \quad p$-tolylboronic acid, ${ }^{7} m$-tolylboronic acid, ${ }^{7} p$ methoxyphenylboronic acid, ${ }^{7}$ and $p$-chlorophenylboronic acid $^{7}$ were prepared following the literature procedures. All other chemicals and solvents were purchased from Aldrich, Wako, TCI, or Kanto Chemicals and used as received.

\section{Rhodium-Catalyzed 1,4-Addition of Arylboronic Acids to Coumarins}

\section{General Procedure}

A solution of $\mathrm{Rh}(\mathrm{acac})\left(\mathrm{C}_{2} \mathrm{H}_{4}\right)_{2}(2.3 \mathrm{mg}, 9.0 \mu \mathrm{mol})$, chiral ligand $(9.9 \mu \mathrm{mol})$, coumarin substrate $(0.30 \mathrm{mmol})$ and $\operatorname{ArB}(\mathrm{OH})_{2}(3.0 \mathrm{mmol})$ in a mixture of $1,4-$ dioxane $(1.0 \mathrm{~mL})$ and $\mathrm{H}_{2} \mathrm{O}(0.10 \mathrm{~mL})$ was stirred at $60{ }^{\circ} \mathrm{C}$ for $8-12 \mathrm{~h}$. After the completion of the reaction, the mixture was passed through a short pad of silica gel with $\mathrm{Et}_{2} \mathrm{O}$ as eluent, and the solvent was removed under reduced pressure. The residue was purified by column chromatography on silica gel with hexane/EtOAc (5/1) as eluent (unless otherwise stated) to afford the desired 1,4-adduct 4.<smiles>Cc1ccc2c(c1)[C@@H](c1ccccc1)CC(=O)O2</smiles>

(R)-6-Methyl-4-phenylchroman-2-one (4am) [CAS: 827007-19-2]

Table 2, entry 1. White solid, $88 \%$ yield, $99.6 \%$ ee. The ee was determined on a Daicel Chiralcel OD-H column with hexane : 2-propanol $=95: 5$, flow $=1.0$ 
$\mathrm{mL} / \mathrm{min}$, wavelength $=254 \mathrm{~nm}$. Retention time: $14.5 \mathrm{~min}[(S)$-enantiomer $], 17.4 \mathrm{~min}$ $\left[(R)\right.$-enantiomer]. $[\alpha]_{\mathrm{D}}^{20}-6.2\left(c 1.00, \mathrm{CHCl}_{3}\right)$. The absolute configuration was determined by conversion into $(R)$-tolterodine L-tartrate and comparison of the optical rotation with literature value. ${ }^{8}$

${ }^{1} \mathrm{H}$ NMR $\left(500 \mathrm{MHz}, \mathrm{CDCl}_{3}\right): \delta 7.35(\mathrm{t}, J=7.3 \mathrm{~Hz}, 2 \mathrm{H}), 7.29(\mathrm{t}, J=7.3 \mathrm{~Hz}, 1 \mathrm{H})$, $7.15(\mathrm{~d}, J=7.4 \mathrm{~Hz}, 2 \mathrm{H}), 7.09(\mathrm{~d}, J=8.3 \mathrm{~Hz}, 1 \mathrm{H}), 7.02(\mathrm{~d}, J=8.3 \mathrm{~Hz}, 1 \mathrm{H}), 6.78(\mathrm{~s}$, $1 \mathrm{H}), 4.29(\mathrm{dd}, J=7.5,6.1 \mathrm{~Hz}, 1 \mathrm{H}), 3.05(\mathrm{dd}, J=15.9,6.1 \mathrm{~Hz}, 1 \mathrm{H}), 3.00(\mathrm{dd}, J=15.9$, $7.5 \mathrm{~Hz}, 1 \mathrm{H}), 2.25(\mathrm{~s}, 3 \mathrm{H}) .{ }^{13} \mathrm{C} \mathrm{NMR}\left(125 \mathrm{MHz}, \mathrm{CDCl}_{3}\right): \delta 167.7,149.6,140.5,134.3$, 129.3, 129.1, 128.6, 127.6, 127.5, 125.3, 116.8, 40.7, 37.1, 20.7. Anal. Calcd for $\mathrm{C}_{16} \mathrm{H}_{14} \mathrm{O}_{2}: \mathrm{C}, 80.65 ; \mathrm{H}, 5.92$. Found: C, 80.51; H, 5.87.<smiles>Cc1ccc([C@H]2CC(=O)Oc3ccc(C)cc32)cc1</smiles>

(R)-6-Methyl-4-(4-methylphenyl)chroman-2-one (4an)

Table 2, entry 2. White solid, $91 \%$ yield, $99.8 \%$ ee. The ee was determined on a Daicel Chiralcel OD-H column with hexane $: 2$-propanol $=95: 5$, flow $=1.0$ $\mathrm{mL} / \mathrm{min}$, wavelength $=254 \mathrm{~nm}$. Retention time: $13.2 \mathrm{~min}[(S)$-enantiomer $], 14.9 \mathrm{~min}$ $[(R)$-enantiomer $] . \quad[\alpha]_{\mathrm{D}}^{20}+1.3\left(c 1.00, \mathrm{CHCl}_{3}\right)$. The absolute configuration was assigned by analogy with Table 2 , entry 1 .

${ }^{1} \mathrm{H}$ NMR $\left(500 \mathrm{MHz}, \mathrm{CDCl}_{3}\right): \delta 7.15(\mathrm{~d}, J=8.3 \mathrm{~Hz}, 2 \mathrm{H}), 7.08(\mathrm{~d}, J=8.3 \mathrm{~Hz}$, $1 \mathrm{H}), 7.04(\mathrm{~d}, J=8.1 \mathrm{~Hz}, 2 \mathrm{H}), 7.01(\mathrm{~d}, J=8.3 \mathrm{~Hz}, 1 \mathrm{H}), 6.78(\mathrm{~s}, 1 \mathrm{H}), 4.25(\mathrm{dd}, J=7.6$, $5.9 \mathrm{~Hz}, 1 \mathrm{H}), 3.03(\mathrm{dd}, J=15.7,5.9 \mathrm{~Hz}, 1 \mathrm{H}), 2.97(\mathrm{dd}, J=15.8,7.6 \mathrm{~Hz}, 1 \mathrm{H}), 2.34(\mathrm{~s}$, $3 \mathrm{H}), 2.25$ (s, 3H). ${ }^{13} \mathrm{C} \mathrm{NMR}\left(125 \mathrm{MHz}, \mathrm{CDCl}_{3}\right): \delta 167.8,149.6,137.4,137.2,134.2$, 129.7, 129.1, 128.6, 127.3, 125.5, 116.7, 40.3, 37.1, 21.0, 20.7. Anal. Calcd for $\mathrm{C}_{17} \mathrm{H}_{16} \mathrm{O}_{2}:$ C, 80.93; H, 6.39. Found: C, 80.97; H, 6.42.<smiles>Cc1cccc([C@H]2CC(=O)Oc3ccc(C)cc32)c1</smiles>

(R)-6-Methyl-4-(3-methylphenyl)chroman-2-one (4ao)

Table 2, entry 3. White solid, $93 \%$ yield, $99.7 \%$ ee. The ee was determined on a Daicel Chiralcel OD-H column with hexane : 2-propanol $=95: 5$, flow $=1.0$ $\mathrm{mL} / \mathrm{min}$, wavelength $=254 \mathrm{~nm}$. Retention time: $13.2 \mathrm{~min}[(S)$-enantiomer], $16.2 \mathrm{~min}$ $[(R)$-enantiomer $] .[\alpha]_{\mathrm{D}}^{20}-6.7\left(c 1.00, \mathrm{CHCl}_{3}\right)$. The absolute configuration was assigned by analogy with Table 2 , entry 1 .

${ }^{1} \mathrm{H}$ NMR $\left(500 \mathrm{MHz}, \mathrm{CDCl}_{3}\right): \delta 7.23(\mathrm{t}, J=7.6 \mathrm{~Hz}, 1 \mathrm{H}), 7.10(\mathrm{~s}, 1 \mathrm{H}), 7.08(\mathrm{~d}, J$ $=8.0 \mathrm{~Hz}, 1 \mathrm{H}), 7.02(\mathrm{~d}, J=8.3 \mathrm{~Hz}, 1 \mathrm{H}), 6.96-6.93(\mathrm{~m}, 2 \mathrm{H}), 6.78(\mathrm{~s}, 1 \mathrm{H}), 4.25(\mathrm{dd}, J=$ 7.4, 6.1 Hz, 1H), $3.03(\mathrm{dd}, J=15.9,6.1 \mathrm{~Hz}, 1 \mathrm{H}), 2.98(\mathrm{dd}, J=15.9,7.4 \mathrm{~Hz}, 1 \mathrm{H}), 2.33$ $(\mathrm{s}, 3 \mathrm{H}), 2.26(\mathrm{~s}, 3 \mathrm{H}) .{ }^{13} \mathrm{C} \mathrm{NMR}\left(125 \mathrm{MHz}, \mathrm{CDCl}_{3}\right): \delta 167.8,149.6,140.5,138.7$, 134.2, 129.2, 128.9, 128.6, 128.3, 128.1, 125.4, 124.5, 116.7, 40.6, 37.0, 21.4, 20.7. Anal. Calcd for $\mathrm{C}_{17} \mathrm{H}_{16} \mathrm{O}_{2}$ : C, 80.93; H, 6.39. Found: C, 81.15; H, 6.45. 
<smiles>Cc1ccc2c(c1)[C@H](c1ccc(Cl)cc1)CC(=O)O2</smiles>

(R)-6-Methyl-4-(4-chlorophenyl)chroman-2-one (4ap) [CAS: 243666-35-5]

Table 2, entry 5. Pale yellow oil, $45 \%$ yield, $99.1 \%$ ee. The ee was determined on a Daicel Chiralcel OD-H column with hexane : 2-propanol $=95: 5$, flow $=1.0 \mathrm{~mL} / \mathrm{min}$, wavelength $=254 \mathrm{~nm}$. Retention time: $18.0 \mathrm{~min}[(S)$ enantiomer $], 19.6 \min [(R)$-enantiomer $] .[\alpha]^{20}{ }_{D}+5.3\left(c 1.00, \mathrm{CHCl}_{3}\right)$. The absolute configuration was assigned by analogy with Table 2 , entry 1 .

${ }^{1} \mathrm{H}$ NMR $\left(500 \mathrm{MHz}, \mathrm{CDCl}_{3}\right): \delta 7.31(\mathrm{~d}, J=8.4 \mathrm{~Hz}, 2 \mathrm{H}), 7.11-7.08(\mathrm{~m}, 3 \mathrm{H})$, $7.02(\mathrm{~d}, J=8.3 \mathrm{~Hz}, 1 \mathrm{H}), 6.77(\mathrm{~s}, 1 \mathrm{H}), 4.27(\mathrm{dd}, J=7.3,6.0 \mathrm{~Hz}, 1 \mathrm{H}), 3.03(\mathrm{dd}, J=$ 15.8, $6.0 \mathrm{~Hz}, 1 \mathrm{H}), 2.95(\mathrm{dd}, J=15.8,7.3 \mathrm{~Hz}, 1 \mathrm{H}), 2.26(\mathrm{~s}, 3 \mathrm{H}) .{ }^{13} \mathrm{C} \mathrm{NMR}(125 \mathrm{MHz}$, $\left.\mathrm{CDCl}_{3}\right): \delta 167.3,149.6,139.0,134.4,133.4,129.5,129.2,128.8,128.5,124.7,116.9$, 40.1, 37.0, 20.7. Anal. Calcd for $\mathrm{C}_{16} \mathrm{H}_{13} \mathrm{ClO}_{2}$ : C, 70.46; H, 4.80. Found: C, 70.73; H, 5.06 .<smiles>COc1ccc([C@H]2CC(=O)Oc3ccc(C)cc32)cc1</smiles>

(R)-6-Methyl-4-(4-methoxyphenyl)chroman-2-one (4aq) [CAS: 243666-35-5]

Table 2, entry 4. Purified by column chromatography on silica gel with dichloromethane as eluent. White solid, $75 \%$ yield, $99.5 \%$ ee. The ee was determined on a Daicel Chiralpak AD-H column with hexane : 2-propanol $=90: 10$, flow $=0.8 \mathrm{~mL} / \mathrm{min}$, wavelength $=254 \mathrm{~nm}$. Retention time: $19.2 \mathrm{~min}[(S)-$ enantiomer], $20.9 \mathrm{~min}[(R)$-enantiomer $] .[\alpha]_{\mathrm{D}}^{20}+5.6\left(c 0.99, \mathrm{CHCl}_{3}\right)$. The absolute configuration was assigned by analogy with Table 2 , entry 1 .

${ }^{1} \mathrm{H}$ NMR $\left(500 \mathrm{MHz}, \mathrm{CDCl}_{3}\right): \delta$ 7.08-7.06 (m, 3H), $7.01(\mathrm{~d}, J=8.3 \mathrm{~Hz}, 1 \mathrm{H}), 6.89$ $6.87(\mathrm{~m}, 2 \mathrm{H}), 6.78(\mathrm{~s}, 1 \mathrm{H}), 4.24(\mathrm{dd}, J=7.7,6.0 \mathrm{~Hz}, 1 \mathrm{H}), 3.02(\mathrm{dd}, J=15.9,6.0 \mathrm{~Hz}$, $1 \mathrm{H}), 2.96(\mathrm{dd}, J=15.9,7.7 \mathrm{~Hz}, 1 \mathrm{H}), 2.26(\mathrm{~s}, 3 \mathrm{H}) .{ }^{13} \mathrm{C} \mathrm{NMR}\left(125 \mathrm{MHz}, \mathrm{CDCl}_{3}\right): \delta$ 167.9, 159.0, 149.6, 134.3, 132.5, 129.2, 128.6, 128.6, 125.8, 116.8, 114.5, 55.3, 40.0, 37.3, 20.8. Anal. Calcd for $\mathrm{C}_{17} \mathrm{H}_{16} \mathrm{O}_{3}$ : C, 76.10; H, 6.01. Found: C, 76.08; H, 5.94.<smiles>O=C1C[C@H](c2ccccc2)c2ccccc2O1</smiles>

(R)-4-Phenylchroman-2-one (4bm) [CAS: 732250-35-0]

Table 2, entry 6. White solid, $86 \%$ yield, $99.4 \%$ ee. The ee was determined on a Daicel Chiralcel OD-H column with hexane $: 2$-propanol $=95: 5$, flow $=1.0$ 
$\mathrm{mL} / \mathrm{min}$, wavelength $=254 \mathrm{~nm}$. Retention time: $16.2 \mathrm{~min}[(S)$-enantiomer $], 17.6 \mathrm{~min}$ $[(R)$-enantiomer $] .[\alpha]_{D}^{20}-45.1\left(c 0.98, \mathrm{CHCl}_{3}\right)$. The absolute configuration was assigned by analogy with Table 2 , entry 1 .

${ }^{1} \mathrm{H}$ NMR (500 MHz, $\left.\mathrm{CDCl}_{3}\right): \delta$ 7.37-7.33 (m, 2H), 7.32-7.27 (m, 2H), 7.17-7.13 $(\mathrm{m}, 3 \mathrm{H}), 7.08(\mathrm{dt}, J=7.4,1.2 \mathrm{~Hz}, 1 \mathrm{H}), 6.98(\mathrm{~d}, J=7.7 \mathrm{~Hz}, 1 \mathrm{H}), 4.35(\mathrm{dd}, J=7.8,6.1$ $\mathrm{Hz}, 1 \mathrm{H}), 3.08(\mathrm{dd}, J=14.3,6.1 \mathrm{~Hz}, 1 \mathrm{H}), 3.03(\mathrm{dd}, J=14.3,7.8 \mathrm{~Hz}, 1 \mathrm{H}) .{ }^{13} \mathrm{C}$ NMR $\left(125 \mathrm{MHz}, \mathrm{CDCl}_{3}\right): \delta 167.5,151.7,140.2,129.1,128.7,128.3,127.6,127.5,125.7$, 124.6, 117.0, 40.6, 36.9.<smiles>Cc1ccc(C2CC(=O)Oc3ccccc32)cc1</smiles>

\section{(R)-4-(4-Methylphenyl)chroman-2-one (4bn)}

Table 2, entry 7. White solid, $89 \%$ yield, $99.7 \%$ ee. The ee was determined on a Daicel Chiralcel OD-H column with hexane : 2-propanol $=95: 5$, flow $=0.8$ $\mathrm{mL} / \mathrm{min}$, wavelength $=254 \mathrm{~nm}$. Retention time: $18.6 \mathrm{~min}[(S)$-enantiomer], $20.2 \mathrm{~min}$ $[(R)$-enantiomer $] .[\alpha]_{\mathrm{D}}^{20}-41.4\left(c 0.99, \mathrm{CHCl}_{3}\right)$. The absolute configuration was assigned by analogy with Table 2 , entry 1 .

${ }^{1} \mathrm{H}$ NMR $\left(500 \mathrm{MHz}, \mathrm{CDCl}_{3}\right): \delta 7.29(\mathrm{~d}, J=7.8,1.7 \mathrm{~Hz}, 1 \mathrm{H}), 7.16-7.11(\mathrm{~m}, 3 \mathrm{H})$, 7.09-7.04 (m, 3H), $6.97(\mathrm{~d}, J=7.6 \mathrm{~Hz}, 1 \mathrm{H}), 4.31(\mathrm{dd}, J=8.0,6.0 \mathrm{~Hz}, 1 \mathrm{H}), 3.05(\mathrm{dd}, J$ $=15.7,6.0 \mathrm{~Hz}, 1 \mathrm{H}), 3.00(\mathrm{dd}, J=15.8,8.0 \mathrm{~Hz}, 1 \mathrm{H}), 2.34(\mathrm{~s}, 3 \mathrm{H}) .{ }^{13} \mathrm{C}$ NMR $(125$ $\left.\mathrm{MHz}, \mathrm{CDCl}_{3}\right): \delta 167.6,151.6,137.3,137.2,129.7,128.6,128.2,127.3,126.0,124.5$, 117.0, 40.2, 37.0, 20.9. Anal. Calcd for $\mathrm{C}_{16} \mathrm{H}_{14} \mathrm{O}_{2}: \mathrm{C}, 80.65 ; \mathrm{H}, 5.92$. Found: $\mathrm{C}$, 80.64; H, 5.98.<smiles>Cc1cccc(C2CC(=O)Oc3ccccc32)c1</smiles>

(R)-4-(3-Methylphenyl)chroman-2-one (4bo)

Table 2, entry 8. White solid, $90 \%$ yield, $98.7 \%$ ee. The ee was determined on a Daicel Chiralcel OD-H column with hexane : 2-propanol $=95: 5$, flow $=0.8$ $\mathrm{mL} / \mathrm{min}$, wavelength $=254 \mathrm{~nm}$. Retention time: $13.0 \mathrm{~min}[(S)$-enantiomer $], 14.3 \mathrm{~min}$ $[(R)$-enantiomer $] .[\alpha]_{D}^{20}-44.6\left(c 0.98, \mathrm{CHCl}_{3}\right)$. The absolute configuration was assigned by analogy with Table 2 , entry 1 .

${ }^{1} \mathrm{H} \mathrm{NMR}\left(500 \mathrm{MHz}, \mathrm{CDCl}_{3}\right): \delta 7.29(\mathrm{t}, J=7.7 \mathrm{~Hz}, 1 \mathrm{H}), 7.23(\mathrm{t}, J=7.5 \mathrm{~Hz}, 1 \mathrm{H})$, 7.14-7.06 (m, 3H), 6.98-6.94 (m, 3H), $4.30(\mathrm{dd}, J=7.9,6.1 \mathrm{~Hz}, 1 \mathrm{H}), 3.06(\mathrm{dd}, J=$ 15.9, 6.1 Hz, 1H), $3.01(\mathrm{dd}, J=15.9,7.9 \mathrm{~Hz}, 1 \mathrm{H}), 2.33(\mathrm{~s}, 3 \mathrm{H}) .{ }^{13} \mathrm{C} \mathrm{NMR}(125 \mathrm{MHz}$, $\left.\mathrm{CDCl}_{3}\right): \delta 167.6,151.6,140.2,138.7,128.9,128.6,128.3,128.3,128.1,125.8,124.5$, 124.5, 117.0, 40.5, 36.9, 21.4. Anal. Calcd for $\mathrm{C}_{16} \mathrm{H}_{14} \mathrm{O}_{2}: \mathrm{C}, 80.65 ; \mathrm{H}$, 5.92. Found: C, 80.59; H, 5.89. 
<smiles>COC(=O)c1ccc2c(c1)[C@@H](c1ccccc1)CC(=O)O2</smiles>

Methyl (R)-2-oxo-4-phenylchromane-6-carboxylate (4cm) [CAS: 380636-42-0]

Table 2, entry 9. Purified by column chromatography on silica gel with hexane/EtOAc (3/1) as eluent. White solid, 94\% yield, $99.3 \%$ ee. The ee was determined on a Daicel Chiralcel OD-H column with hexane : 2-propanol $=2: 1$, flow $=0.6 \mathrm{~mL} / \mathrm{min}$, wavelength $=254 \mathrm{~nm}$. Retention time: $19.3 \mathrm{~min}[(S)$ enantiomer], $21.3 \mathrm{~min}[(R)$-enantiomer $] .[\alpha]_{\mathrm{D}}^{20}+51.8\left(c 1.00, \mathrm{CHCl}_{3}\right)$. The absolute configuration was assigned by analogy with Table 2 , entry 1 .

${ }^{1} \mathrm{H}$ NMR $\left(500 \mathrm{MHz}, \mathrm{CDCl}_{3}\right): \delta 8.00(\mathrm{dd}, J=8.5,2.0 \mathrm{~Hz}, 1 \mathrm{H}), 7.74(\mathrm{~d}, J=2.0$ $\mathrm{Hz}, 1 \mathrm{H}), 7.35(\mathrm{t}, J=7.2 \mathrm{~Hz}, 2 \mathrm{H}), 7.30(\mathrm{t}, J=7.3 \mathrm{~Hz}, 1 \mathrm{H}), 7.18(\mathrm{~d}, J=8.5 \mathrm{~Hz}, 1 \mathrm{H})$, $7.14(\mathrm{~d}, J=7.1 \mathrm{~Hz}, 2 \mathrm{H}), 4.39(\mathrm{dd}, J=7.2,6.2 \mathrm{~Hz}, 1 \mathrm{H}), 3.86(\mathrm{~s}, 3 \mathrm{H}), 3.10(\mathrm{dd}, J=$ 16.0, $6.2 \mathrm{~Hz}, 1 \mathrm{H}), 3.06(\mathrm{dd}, J=16.0,7.2 \mathrm{~Hz}, 1 \mathrm{H}) .{ }^{13} \mathrm{C} \mathrm{NMR}\left(125 \mathrm{MHz}, \mathrm{CDCl}_{3}\right): \delta$ $166.5,165.9,155.0,139.7,130.5,130.1,129.2$, 127.8, 127.3, 126.6, 125.7, 117.2, $52.1,40.5,36.6$.<smiles>COc1ccc2c(c1)OC(=O)C[C@H]2c1ccccc1</smiles>

(R)-7-Methoxy-4-phenylchroman-2-one (4dm) [CAS: 109475-18-5]

Table 2, entry 10. Purified by column chromatography on silica gel with dichloromethane as eluent. White solid, $28 \%$ yield. $[\alpha]_{\mathrm{D}}^{20}+21.8\left(\mathrm{c} 0.97, \mathrm{CHCl}_{3}\right)$. The absolute configuration was assigned by analogy with Table 2 , entry 1 .

${ }^{1} \mathrm{H} \mathrm{NMR}\left(500 \mathrm{MHz}, \mathrm{CDCl}_{3}\right): \delta 7.34(\mathrm{t}, J=7.4 \mathrm{~Hz}, 2 \mathrm{H}), 7.28(\mathrm{t}, J=7.4 \mathrm{~Hz}, 1 \mathrm{H})$, $7.15(\mathrm{~d}, J=7.0 \mathrm{~Hz}, 2 \mathrm{H}), 6.86(\mathrm{~d}, J=7.7 \mathrm{~Hz}, 1 \mathrm{H}), 6.68(\mathrm{~d}, J=2.6 \mathrm{~Hz}, 1 \mathrm{H}), 6.63(\mathrm{dd}, J$ $=8.4,2.5 \mathrm{~Hz}, 1 \mathrm{H}), 4.28(\mathrm{dd}, J=7.8,6.0 \mathrm{~Hz}, 1 \mathrm{H}), 3.80(\mathrm{~s}, 3 \mathrm{H}), 3.05(\mathrm{dd}, J=15.7,6.0$ $\mathrm{Hz}, 1 \mathrm{H}), 2.99(\mathrm{dd}, J=15.8,7.8 \mathrm{~Hz}, 1 \mathrm{H}) .{ }^{13} \mathrm{C} \mathrm{NMR}\left(125 \mathrm{MHz}, \mathrm{CDCl}_{3}\right): \delta 167.6$, $160.0,152.5,140.8,129.1,128.9,127.6,127.5,117.6,110.7,102.5,55.5,40.1,37.3$.

\section{Rhodium-Catalyzed 1,4-Addition of Phenylboronic Acid to Methyl (E)-3-(2-methoxy-5-methylphenyl)propenoate}<smiles>COc1ccc(OC)c(/C=C/C(C)=O)c1</smiles>

\section{Methyl (E)-3-(2-methoxy-5-methylphenyl)propenoate (6) [CAS:86761-35-5]}

The title compound was synthesized following the literature procedure. ${ }^{6}$ Pale yellow oil. ${ }^{1} \mathrm{H}$ NMR $\left(500 \mathrm{MHz}, \mathrm{CDCl}_{3}\right): \delta 7.97(\mathrm{~d}, J=16.1 \mathrm{~Hz}, 1 \mathrm{H}), 7.31(\mathrm{~s}, 1 \mathrm{H})$, $7.14(\mathrm{~d}, J=8.3 \mathrm{~Hz}, 1 \mathrm{H}), 6.81(\mathrm{~d}, J=8.4 \mathrm{~Hz}, 1 \mathrm{H}), 6.51(\mathrm{~d}, J=16.1 \mathrm{~Hz}, 1 \mathrm{H}), 3.85(\mathrm{~s}$, $3 \mathrm{H}), 3.79(\mathrm{~s}, 3 \mathrm{H}), 2.29(\mathrm{~s}, 3 \mathrm{H}) .{ }^{13} \mathrm{C} \mathrm{NMR}\left(125 \mathrm{MHz}, \mathrm{CDCl}_{3}\right): \delta 167.8,156.3,140.2$, $131.9,129.7,129.2,122.8,117.9,111.0,55.4,51.4,20.2$. 
<smiles>COc1ccc(OC)c(C(CC(C)=O)c2ccccc2)c1</smiles>

Methyl (S)-3-(2-methoxy-5-methylphenyl)-3-phenylpropanoate ((S)-7)

Compound $(S)-7$ in Scheme 3. [CAS: 124937-62-8]

A solution of $\mathrm{Rh}(\mathrm{acac})\left(\mathrm{C}_{2} \mathrm{H}_{4}\right)_{2}(2.3 \mathrm{mg}, 9.0 \mu \mathrm{mol})$, chiral ligand $(9.9 \mu \mathrm{mol})$, methyl (E)-3-(2-methoxy-5-methylphenyl)propenoate $(61.9 \mathrm{mg}, 0.30 \mathrm{mmol})$ and $\mathrm{PhB}(\mathrm{OH})_{2}(366 \mathrm{mg}, 3.0 \mathrm{mmol})$ in a mixture of 1,4-dioxane $(1.0 \mathrm{~mL})$ and $\mathrm{H}_{2} \mathrm{O}(0.10$ $\mathrm{mL}$ ) was stirred at $60{ }^{\circ} \mathrm{C}$ for $10 \mathrm{~h}$. The mixture was passed through a short pad of silica gel with $\mathrm{Et}_{2} \mathrm{O}$ as eluent, and the solvent was removed under reduced pressure. The residue was purified by column chromatography on silica gel with dichloromethane as eluent to afford the desired 1,4-adduct 7.

Colorless oil. $52 \%$ yield, $45 \%$ ee with $(R)$-Segphos as chiral ligand; $43 \%$ yield, $53 \%$ ee with $(R)$-Binap as ligand. The yield was determined by NMR. The ee was determined on a Daicel Chiralcel OD-H column with hexane : 2-propanol $=95: 5$, flow $=0.5 \mathrm{~mL} / \mathrm{min}$, wavelength $=254 \mathrm{~nm}$. Retention time: $12.5 \mathrm{~min}[(S)-$ enantiomer], 13.3 min $[(R)$-enantiomer $] .[\alpha]^{20}{ }_{\mathrm{D}}-0.7\left(c 1.00, \mathrm{CHCl}_{3}, 45 \%\right.$ ee $)$. The absolute configuration was determined by comparison of the optical rotation with that of the $R$-enantiomer, which is derived from $(R)-\mathbf{4 a m}$ by the following procedure.

${ }^{1} \mathrm{H}$ NMR $\left(500 \mathrm{MHz}, \mathrm{CDCl}_{3}\right): \delta$ 7.23-7.27 (m, 4H), $7.15(\mathrm{~m}, 1 \mathrm{H}), 6.96(\mathrm{~d}, J=8.2$ $\mathrm{Hz}, 1 \mathrm{H}), 6.92(\mathrm{~s}, 1 \mathrm{H}), 6.73(\mathrm{~d}, J=8.3 \mathrm{~Hz}, 1 \mathrm{H}), 4.90(\mathrm{t}, J=8.0 \mathrm{~Hz}, 1 \mathrm{H}), 3.74(\mathrm{~s}, 3 \mathrm{H})$, $3.57(\mathrm{~s}, 3 \mathrm{H}), 3.04(\mathrm{~d}, J=8.1 \mathrm{~Hz}, 2 \mathrm{H}), 2.24(\mathrm{~s}, 3 \mathrm{H}) .{ }^{13} \mathrm{C} \mathrm{NMR}\left(125 \mathrm{MHz}, \mathrm{CDCl}_{3}\right): \delta$ 172.6, 154.8, 143.3, 131.7, 129.6, 128.5, 128.2, 127.8, 126.1, 110.9, 55.6, 51.5, 40.4, 39.4, 20.7.<smiles>COc1ccc(OC)c(C(CC(C)=O)c2ccccc2)c1</smiles>

Methyl $(R)-3-(2-m e t h o x y-5-m e t h y l p h e n y l)-3-p h e n y l p r o p a n o a t e ~((R)-7)$ [CAS: 124937-62-8]

$(R)$-6-Methyl-4-phenylchroman-2-one $((R)-4 a m)(99.6 \%$ ee, $80 \mathrm{mg}, 0.34 \mathrm{~mol})$ in a mixture of $\mathrm{MeOH}(0.8 \mathrm{~mL})$ and aqueous sodium hydroxide solution $(2 \mathrm{~N}, 0.8 \mathrm{ml})$ was heated at $70-80{ }^{\circ} \mathrm{C}$ for $1 \mathrm{~h}$. The solution was cooled to $25-30{ }^{\circ} \mathrm{C}$ and $\mathrm{CH}_{2} \mathrm{Cl}_{2}(5$ $\mathrm{mL})$, dimethyl sulfate $(0.20 \mathrm{~mL}, 2.1 \mathrm{mmol})$, tetrabutylammonium bromide $(10 \mathrm{mg})$ was added. The reaction mixture was further stirred at $40^{\circ} \mathrm{C}$ for $10 \mathrm{~h}$. The organic layer was separated, dried over anhydrous magnesium sulfate, chromatographed through a short column of silical gel with $\mathrm{CH}_{2} \mathrm{Cl}_{2}$ as eluent and evaporated to give the titled compound as viscous oil.

Colorless oil. $96 \%$ yield, $95 \%$ ee. The ee was determined on a Daicel Chiralcel OD-H column with hexane $: 2$-propanol $=95: 5$, flow $=0.5 \mathrm{~mL} / \mathrm{min}$, wavelength $=$ $254 \mathrm{~nm}$. Retention time: $12.5 \mathrm{~min}[(S)$-enantiomer], $13.3 \mathrm{~min}[(R)$-enantiomer]. $[\alpha]^{20}+0.8\left(c 1.00, \mathrm{CHCl}_{3}\right) .{ }^{1} \mathrm{H}$ NMR, ${ }^{13} \mathrm{C}$ NMR spectra are identical to those of its $(S)$-enantiomer (see above). 


\section{Asymmetric Synthesis of $(R)$-Tolterodine}<smiles>Cc1ccc2c(c1)[C@@H](c1ccccc1)CC(O)O2</smiles>

\section{(4R)-6-Methyl-4-phenylchroman-2-ol (5) [CAS: 828933-86-4]}

The title compound was synthesized according to the literature procedure. ${ }^{7}(R)$ 6-Methyl-4-phenylchroman-2-one (4am, $238 \mathrm{mg}, 1.00 \mathrm{mmol}$ ) was placed in a Schlenk tube and purged alternately with vacuum and nitrogen. Toluene $(2.0 \mathrm{~mL})$ was added with stirring, and the mixture was cooled to $-20^{\circ} \mathrm{C}$ with an ice-salt bath. Diisobutylaluminum hydride in hexane solution (DIBAL, $0.95 \mathrm{M}, 1.1 \mathrm{~mL}, 1.05$ mmol) was added in portions over $2 \mathrm{~h}$ with syringe while maintaining the reaction temperature at -20 to $-25{ }^{\circ} \mathrm{C}$. The mixture was stirred for an additional $0.5 \mathrm{~h}$, and 0.1 $\mathrm{mL}$ of ethyl acetate was added at -20 to $-25{ }^{\circ} \mathrm{C}$. Aqueous citric acid $(23 \%, 1.0 \mathrm{~mL})$ was added, and the mixture was stirred overnight at $20-25^{\circ} \mathrm{C}$. The organic phase was separated, washed with water $(3 \times 2 \mathrm{~mL})$ and concentrated to half under reduced pressure. Methanol $(2.0 \mathrm{~mL})$ was added, and the solution was concentrated to half again. The methanol addition and evaporation was repeated 3 times to give the title compound in methanol solution. The solvent was evaporated under reduced pressure to give the crude product. Further purification by column chromatography on silica gel $(5 \mathrm{~g})$ with dichloromethane as eluent afforded the title compound as a mixture of diastereomers (white solid, $228 \mathrm{mg}$, $95 \%$ yield).

${ }^{1} \mathrm{H} \mathrm{NMR}\left(500 \mathrm{MHz}, \mathrm{CDCl}_{3}\right): \delta 7.33(\mathrm{t}, J=7.6 \mathrm{~Hz}, 2 \mathrm{H}), 7.26(\mathrm{t}, J=6.0 \mathrm{~Hz}, 1 \mathrm{H})$, 7.20 (t, $J=7.4 \mathrm{~Hz}, 2 \mathrm{H}), 6.94(\mathrm{~d}, J=8.2 \mathrm{~Hz}, 2 \mathrm{H}), 6.81-6.77(\mathrm{~m}, 1 \mathrm{H}), 6.58(\mathrm{~s}, 0.79 \mathrm{H})$, $6.54(\mathrm{~s}, 0.21 \mathrm{H}), 5.64(\mathrm{~d}, J=2.7 \mathrm{~Hz}, 0.79 \mathrm{H}), 5.52(\mathrm{~m}, 0.21 \mathrm{H}), 4.30(\mathrm{dd}, J=10.8 \mathrm{~Hz}, J$ $=5.7 \mathrm{~Hz}, 0.79 \mathrm{H}), 4.19(\mathrm{dd}, J=10.9 \mathrm{~Hz}, J=5.8 \mathrm{~Hz}, 0.21 \mathrm{H}), 2.47-2.43(\mathrm{~m}, 0.21 \mathrm{H})$, 2.28-2.23 (m, 0.8H), 2.17-2.10 (m, 1H), 2.14 (s, 3H).

\section{(R)-Tolterodine (1) [CAS: 124937-51-5]}

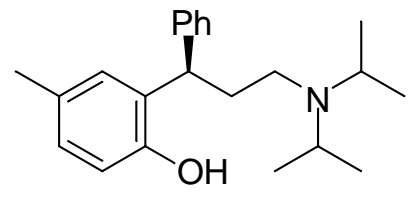

$(R)$-Tolterodine $(\mathbf{1})$ was synthesized following the reported procedure. ${ }^{8}(4 R)-6$ Methyl-4-phenylchroman-2-ol (5, $200 \mathrm{mg}, 0.83 \mathrm{mmol})$ and Pd-C (10\%) (8.8 mg Pd, $0.083 \mathrm{mmol}$ ) were placed in a $50-\mathrm{mL}$ autoclave with a glass inner. The autoclave was purged alternately with vacuum and nitrogen. Methanol $(1.0 \mathrm{~mL})$ and anhydrous diisopropylamine $(0.30 \mathrm{~mL}, 2.1 \mathrm{mmol})$ were added with a syringe. The autoclave was purged with $\mathrm{H}_{2}$ and finally charged with $50 \mathrm{psi} \mathrm{H}_{2}$. The autoclave was placed into a $50{ }^{\circ} \mathrm{C}$ oil bath and the reaction mixture was stirred with a magnetic stirring bar for 12 h. After the completion of the reaction, the autoclave was cooled to room temperature and the residual gas was released. The reaction mixture was filtered through a pad of celite, rinsed with methanol. The solvent was distilled off under reduced pressure. The remaining oil was purified by column chromatography on silica gel with hexane/EtOAc/ $/ \mathrm{Et}_{3} \mathrm{~N}=70 / 30 / 2$. Concentration under reduced pressure yielded $(R)$ tolterodine (1) as colorless oil (247 mg, 91\% yield). $[\alpha]^{20}{ }_{\mathrm{D}}+31.1\left(c 1.00, \mathrm{CH}_{3} \mathrm{OH}\right)$. The absolute configuration was determined by comparison of the optical rotation with that reported in the literature ${ }^{8}$ (Lit. value: $[\alpha]^{20}{ }_{D}-23\left(c\right.$ 1.5, $\left.\mathrm{CH}_{3} \mathrm{OH}\right)$ for $(S)$ - 
enantiomer).

${ }^{1} \mathrm{H}$ NMR $\left(500 \mathrm{MHz}, \mathrm{CDCl}_{3}\right): \delta$ 7.32-7.29 $(\mathrm{m}, 4 \mathrm{H}), 7.23-7.19(\mathrm{~m}, 1 \mathrm{H}), 6.84(\mathrm{dd}$, $J=8.2,2.0 \mathrm{~Hz}, 1 \mathrm{H}), 6.80(\mathrm{~d}, J=8.1 \mathrm{~Hz}, 1 \mathrm{H}), 6.54(\mathrm{~s}, 1 \mathrm{H}), 4.48(\mathrm{dd}, J=10.8,3.8 \mathrm{~Hz}$, $1 \mathrm{H}), 3.21(\mathrm{q}, J=6.7 \mathrm{~Hz}, 2 \mathrm{H}), 2.72-2.69(\mathrm{~m}, 1 \mathrm{H}), 2.40-2.30(\mathrm{~m}, 2 \mathrm{H}), 2.11(\mathrm{~s}, 3 \mathrm{H})$, 2.11-2.04 (m, 1H), $1.12(\mathrm{~d}, J=6.7 \mathrm{~Hz}, 6 \mathrm{H}), 1.06(\mathrm{~d}, J=6.7 \mathrm{~Hz}, 6 \mathrm{H}) .{ }^{13} \mathrm{C}$ NMR $(125$ $\left.\mathrm{MHz}, \mathrm{CDCl}_{3}\right): \delta 153.0,144.7,132.3,129.1,128.5,128.3,128.2,127.6,125.9,117.8$, $47.9,42.2,39.5,33.5,20.6,19.9,19.5$.

\section{(R)-Tolterodine L-tartrate [CAS: 124937-51-5]}

L-Tartaric acid (103 $\mathrm{mg}, 0.68 \mathrm{mmol})$ was added to a solution of $(R)$-tolterodine (221 mg, $0.68 \mathrm{mmol})$ in absolute $\mathrm{EtOH}(10 \mathrm{~mL})$ at $80{ }^{\circ} \mathrm{C}$. The mixture was refluxed for additional $0.5 \mathrm{~h}$, gradually cooled down, placed at room temperature overnight, and held at $0{ }^{\circ} \mathrm{C}$ for $1 \mathrm{~h}$. The $(R)$-Tolterodine L-tartrate was collected by filtration, washed with cool EtOH $(10 \mathrm{~mL})$, and dried in vacuo to give the title compound as needle-like white crystal $(272 \mathrm{mg}$, yield $84 \%)$. $[\alpha]^{25}+28.4\left(c 1.00, \mathrm{CH}_{3} \mathrm{OH}\right)$, in accord with that reported in literature (lit. value: $[\alpha]_{D}^{25}+29.1\left(c 1, \mathrm{CH}_{3} \mathrm{OH}\right)$ ).

\section{References:}

(1) Saito, T.; Yokozawa, T.; Ishizaki, T.; Moroi, T.; Sayo, N.; Miura, T.; Kumobayashi, H. Adv. Synth. Catal. 2001, 343, 264.

(2) Pai, C.-C.; Lin, C.-W.; Lin, C.-C.; Chen, C.-C.; Chan, A. S. C.; Wong, W. T. J. Am. Chem. Soc. 2000, 122, 11513.

(3) Takaya, H.; Mashima, K.; Koyano, K.; Yagi, M.; Kumobayashi, H.; Taketomi, T.; Akutagawa, S.; Noyori, R. J. Org. Chem. 1986, 51, 629.

(4) Cramer, R. Inorg. Synth. 1974, 15, 14.

(5) Harayama, T.; Nakatsuka, K.; Nishioka, H.; Murakami, K.; Hayashida, N.; Ishii, H. Chem. Pharm. Bull. 1994, 42, 2170.

(6) Peterson, J. R.; Russell, M. E.; Surjasasmita, I. B. J. Chem. Eng. Data 1988, 33, 534.

(7) Sullivan, J. M.; Barnes, H. H. WO Patent 9964428, 1999.

(8) (a) Gage, J. R.; Cabaj, J. E. US Patent 5,922,914, 1999. (b) Botteghi, C.; Corrias, T.; Marchetti, M. Paganelli, S.; Piccolo, O. Org. Proc. Res. Dev. 2002, 6, 379.

(9) Piccolo, O.; Ulgheri, F.; Marchetti, M. WO Patent 2005005356, 2005. 


\section{V. ${ }^{1}$ H NMR Spectra}
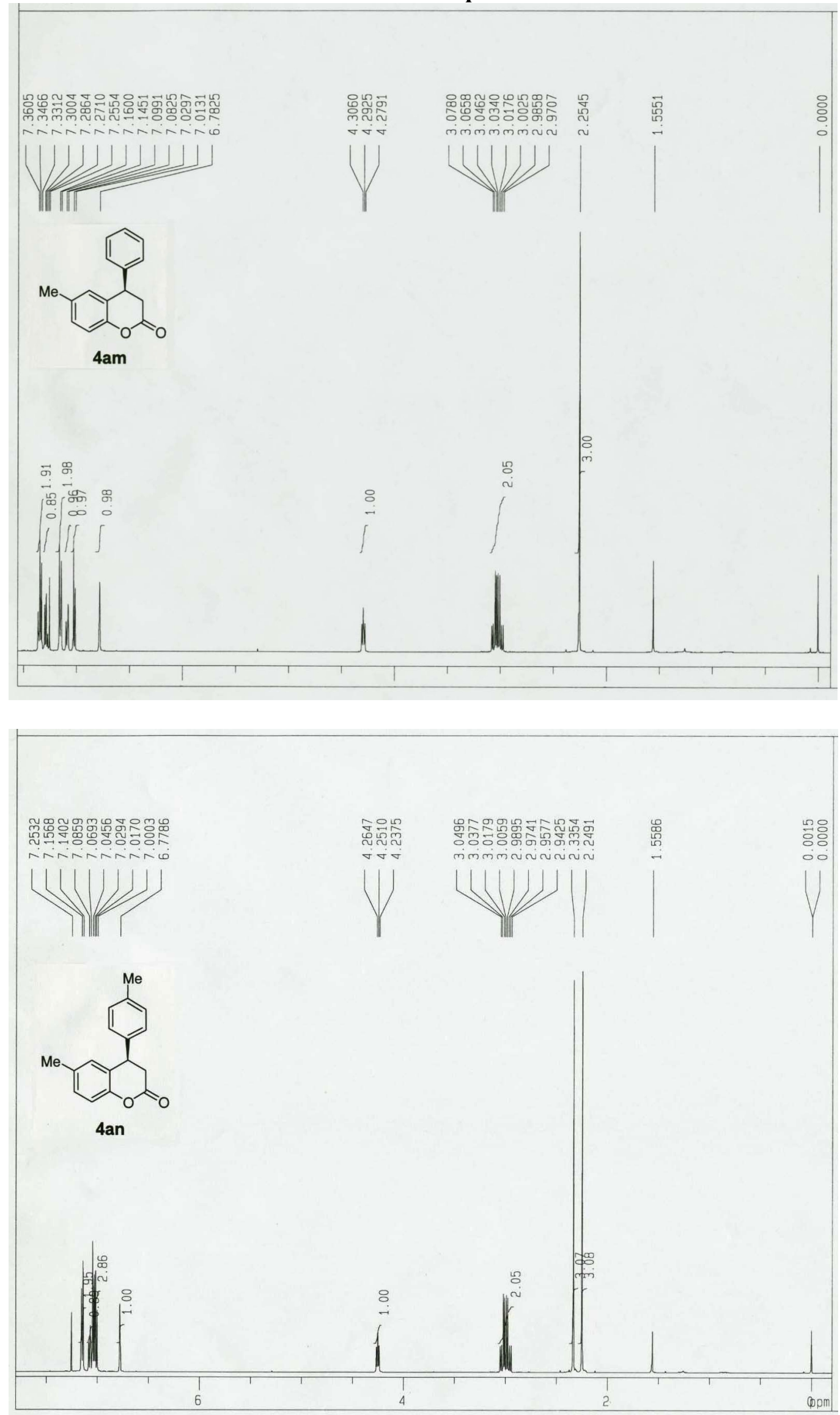

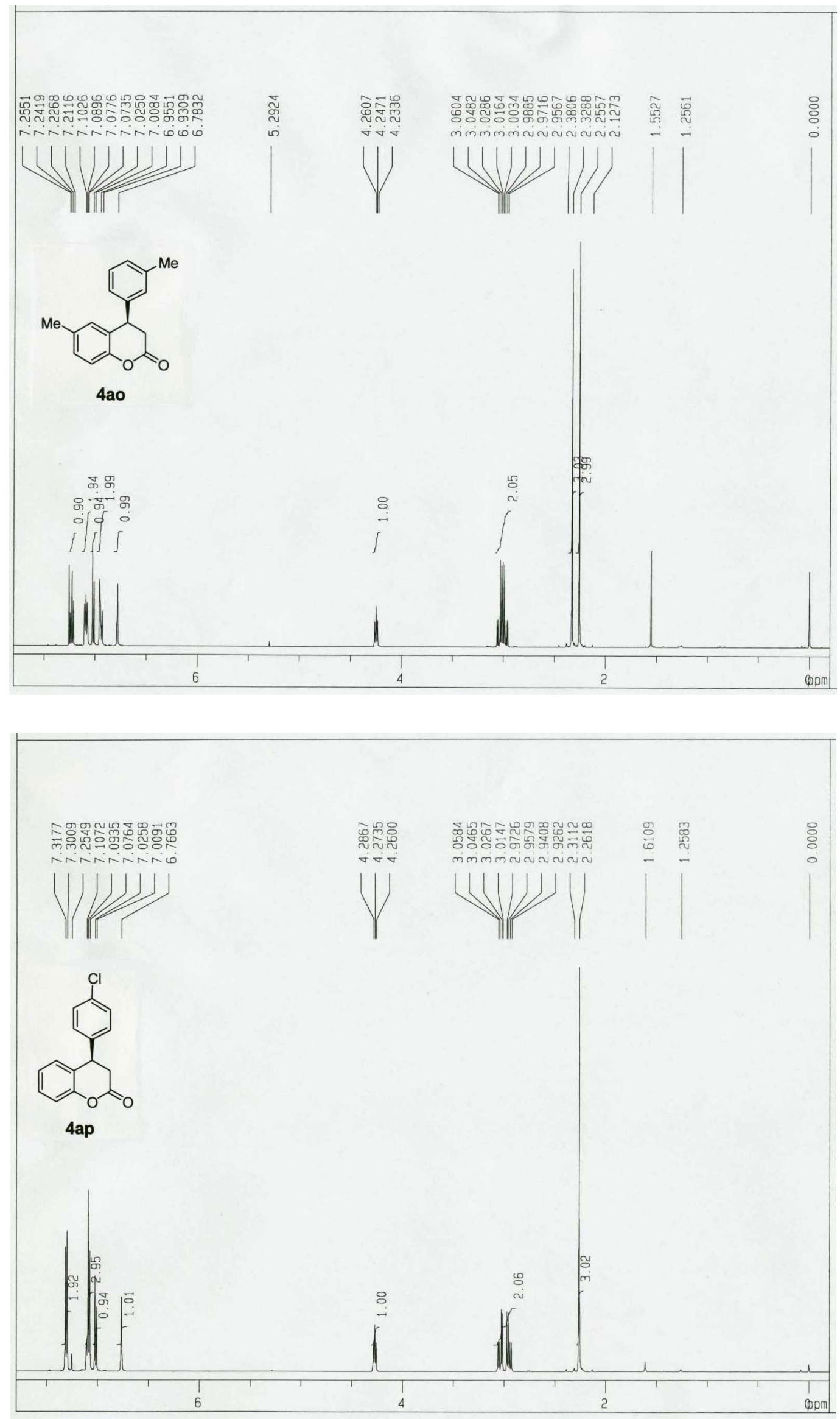

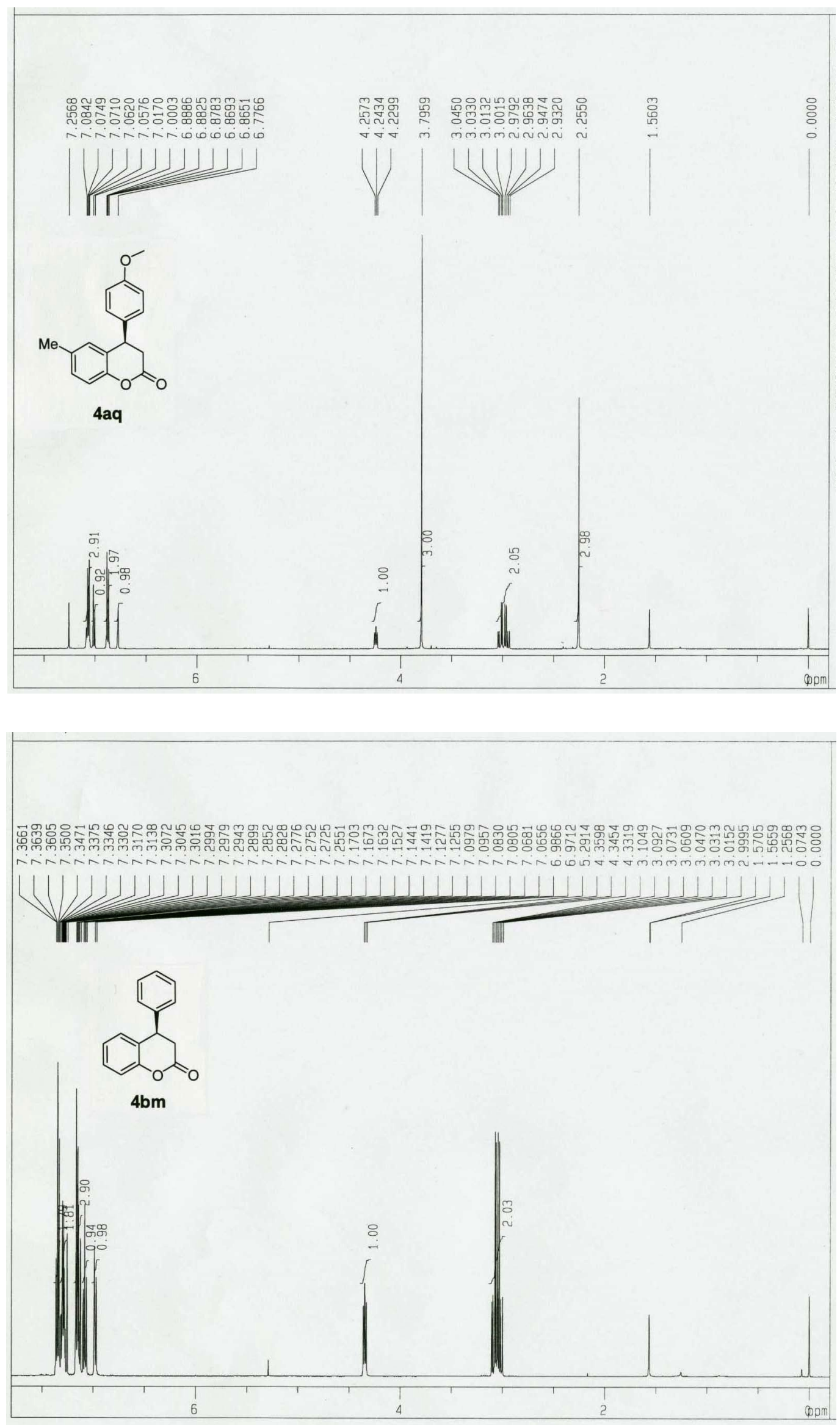

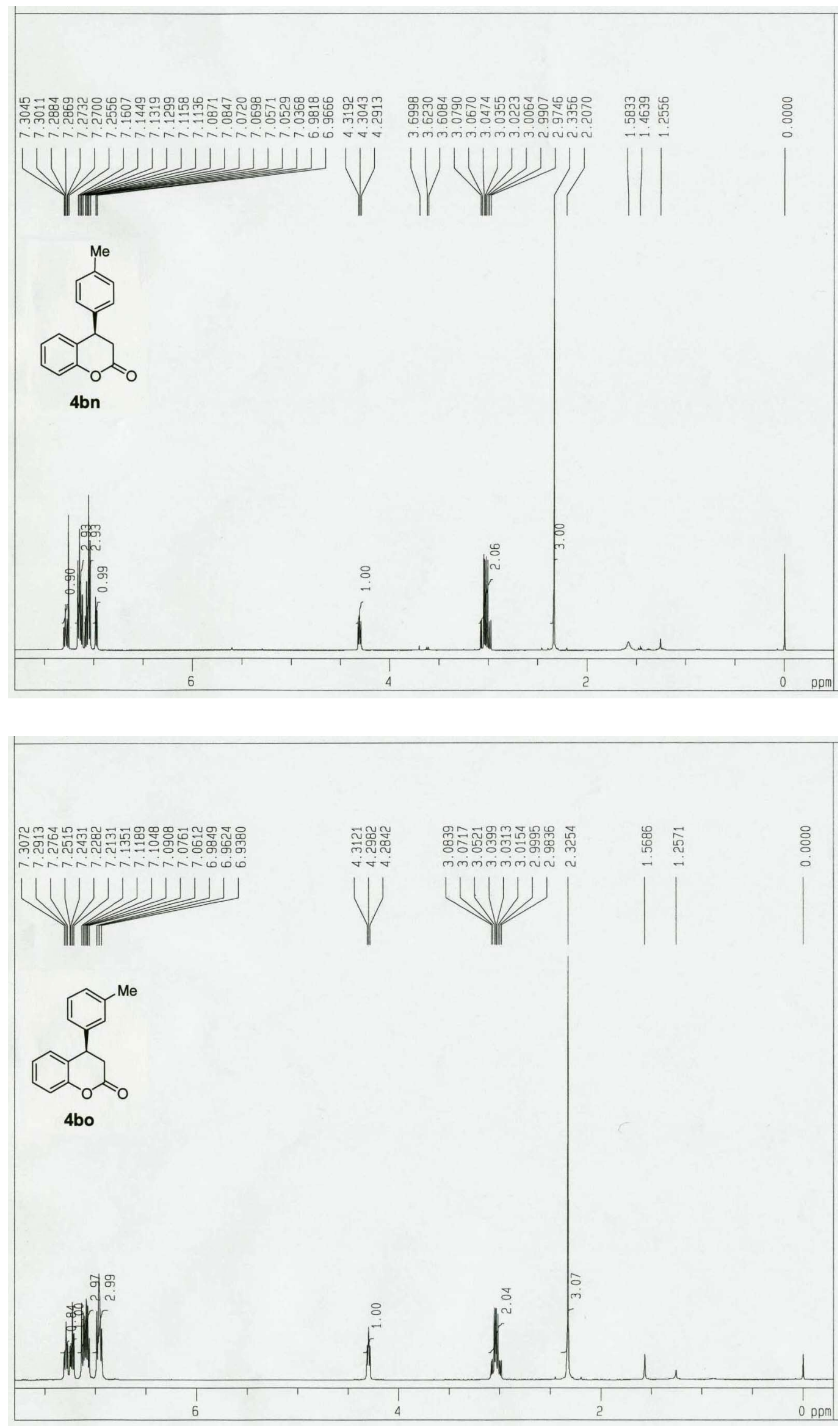

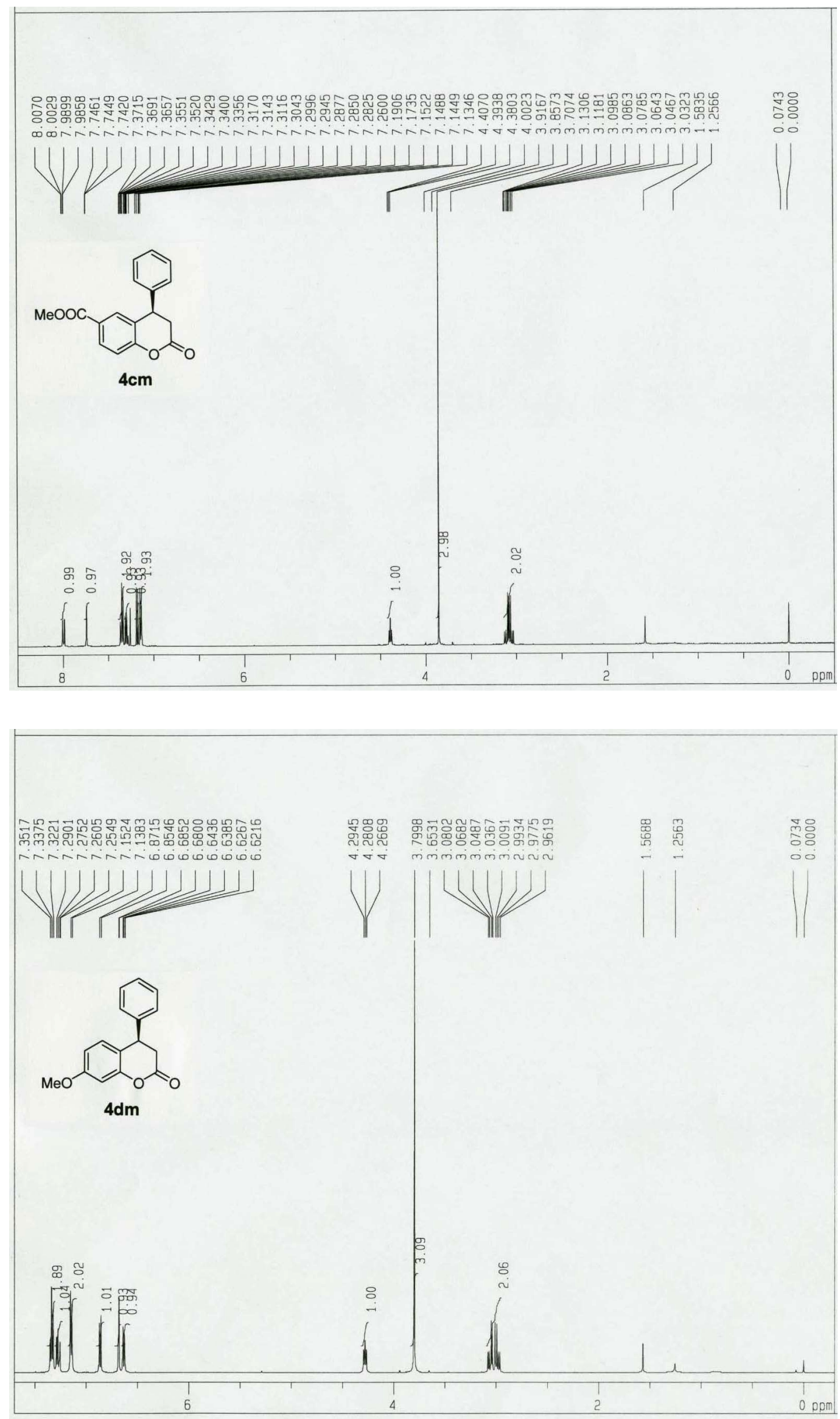

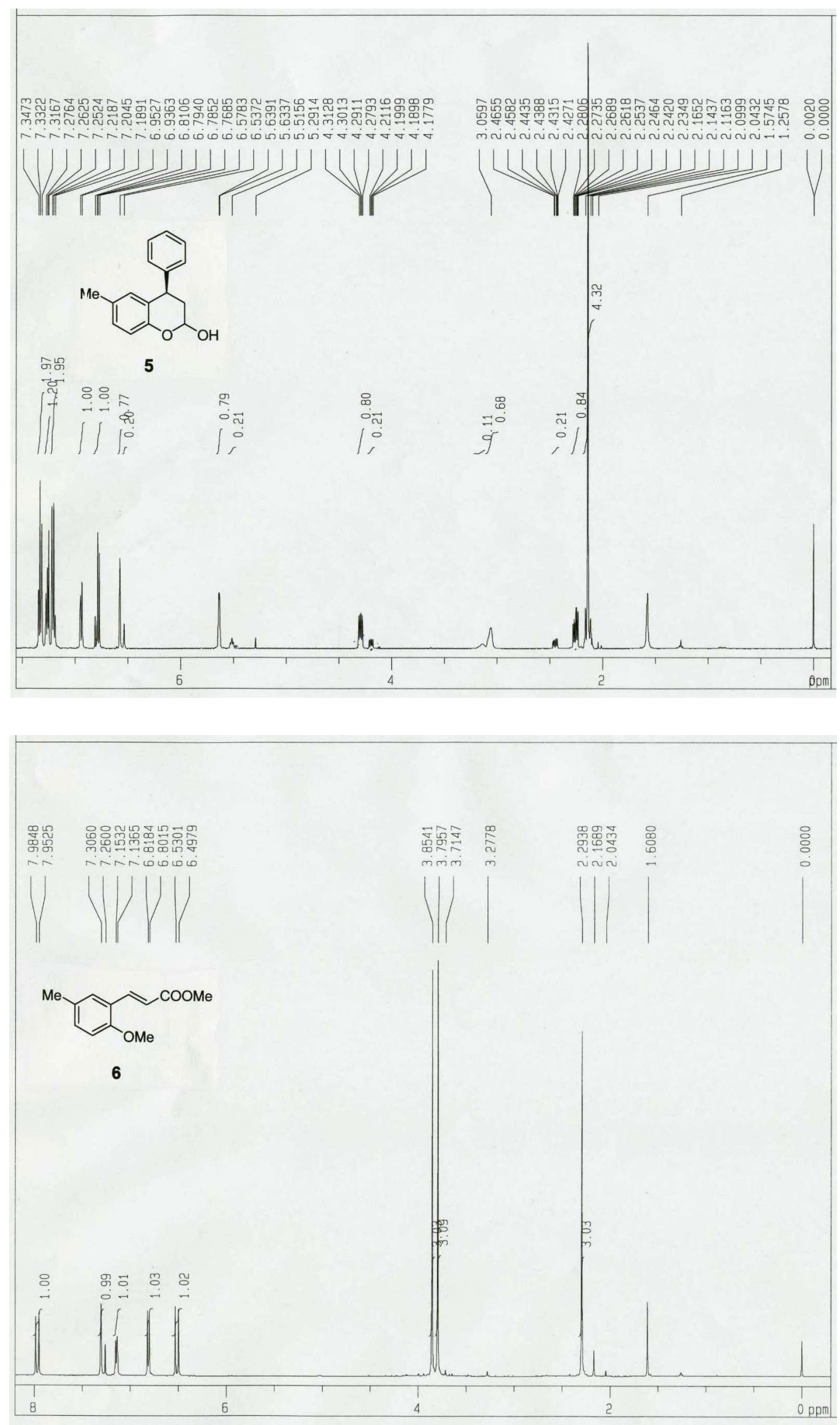

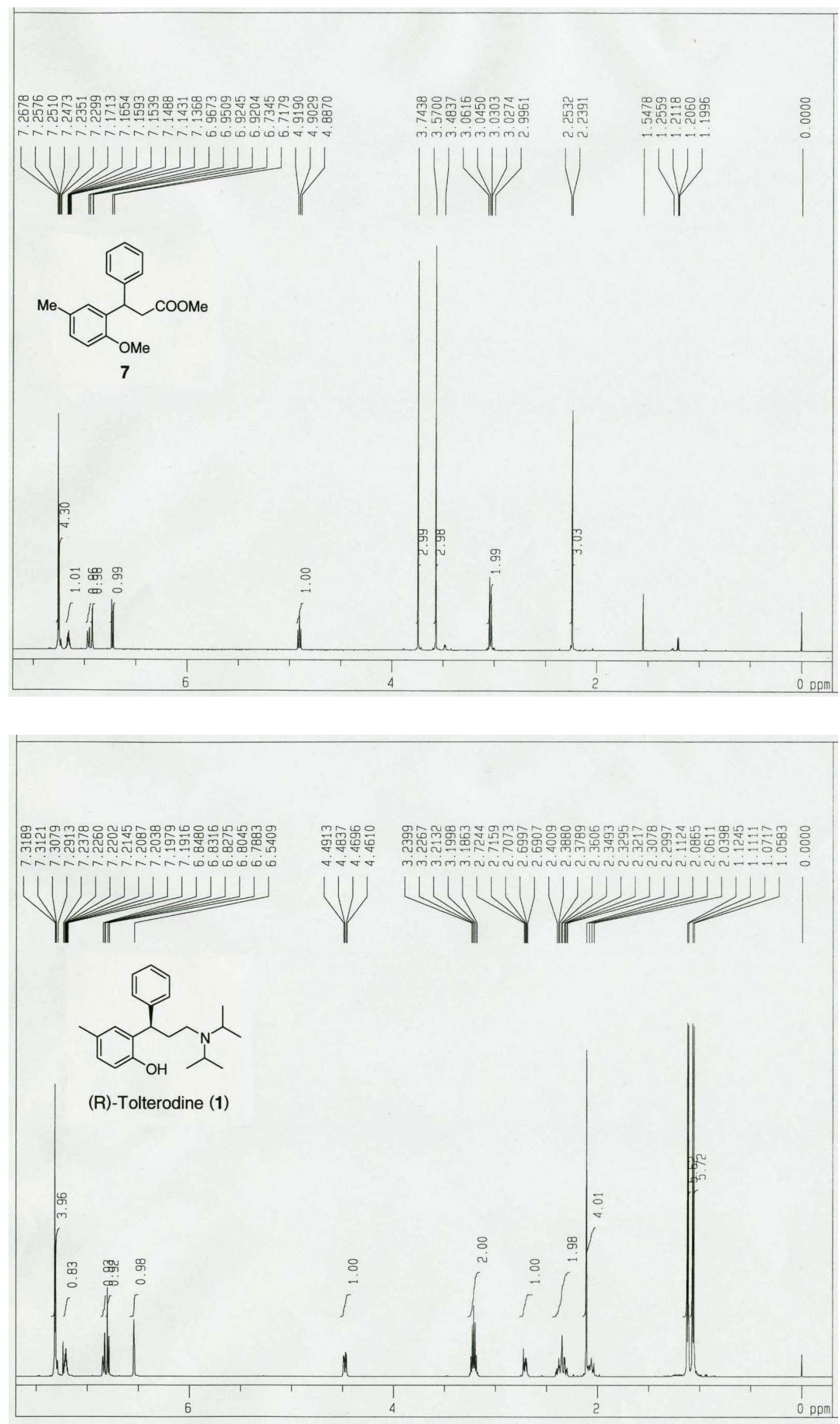

VI. ${ }^{13}$ C NMR Spectra 

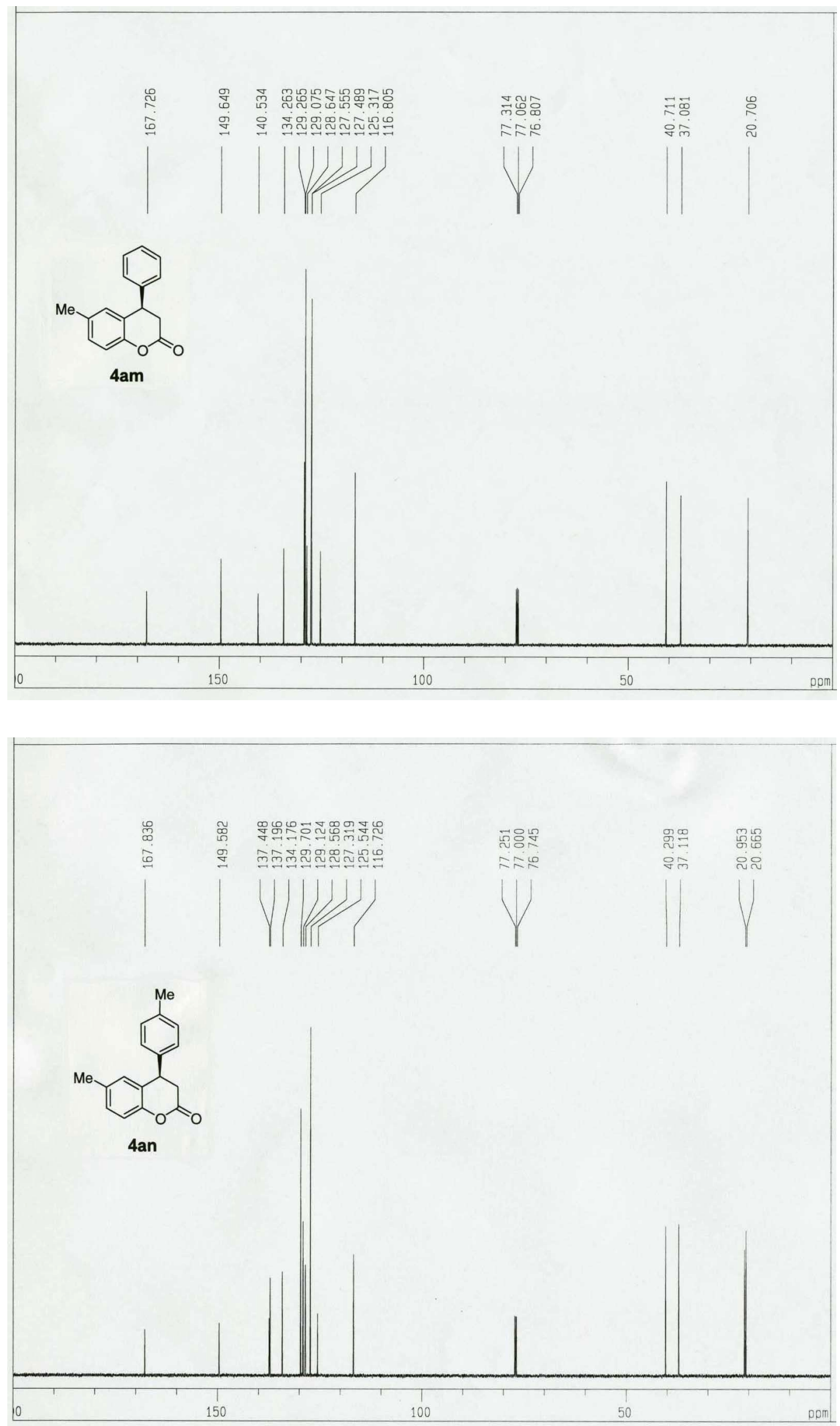

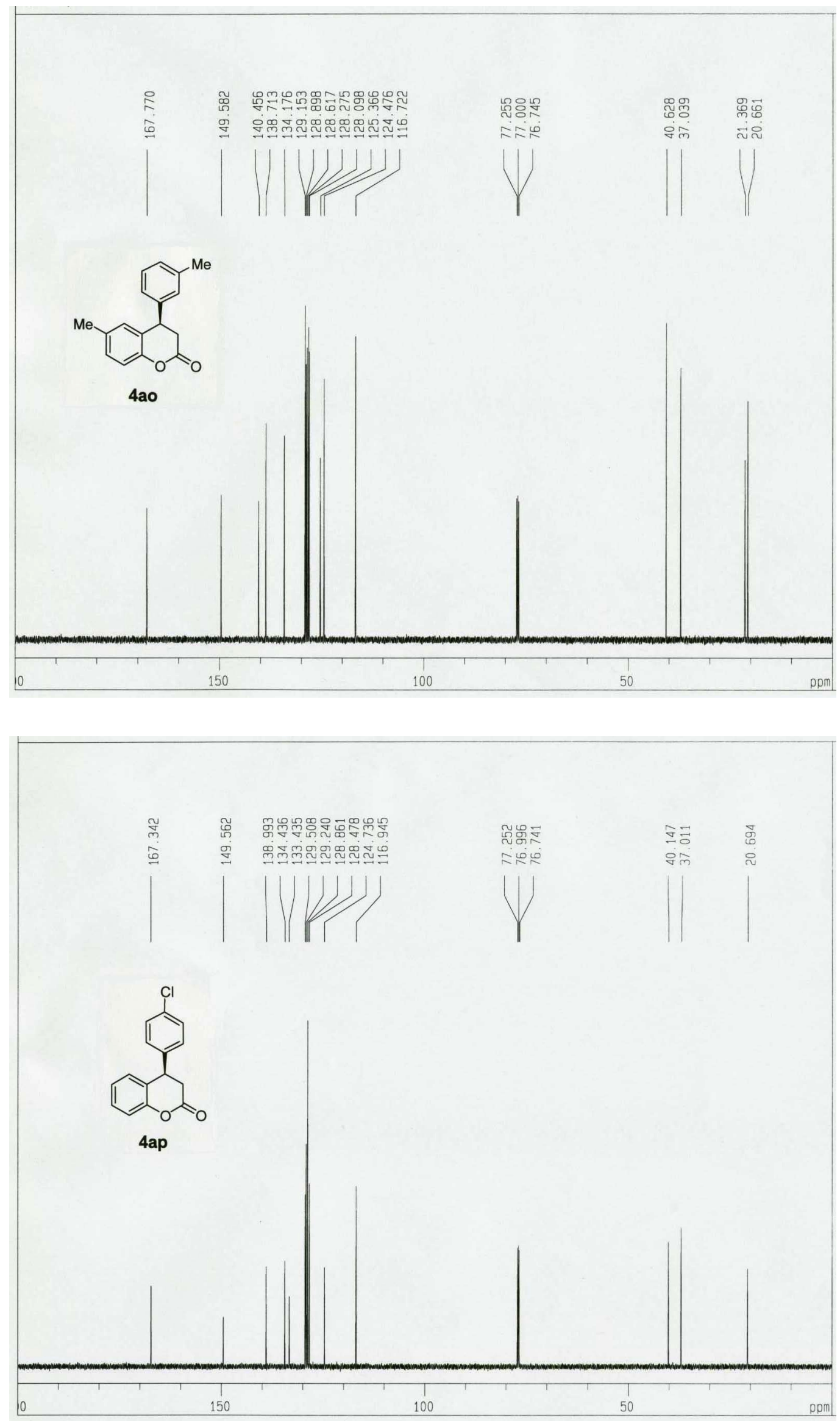

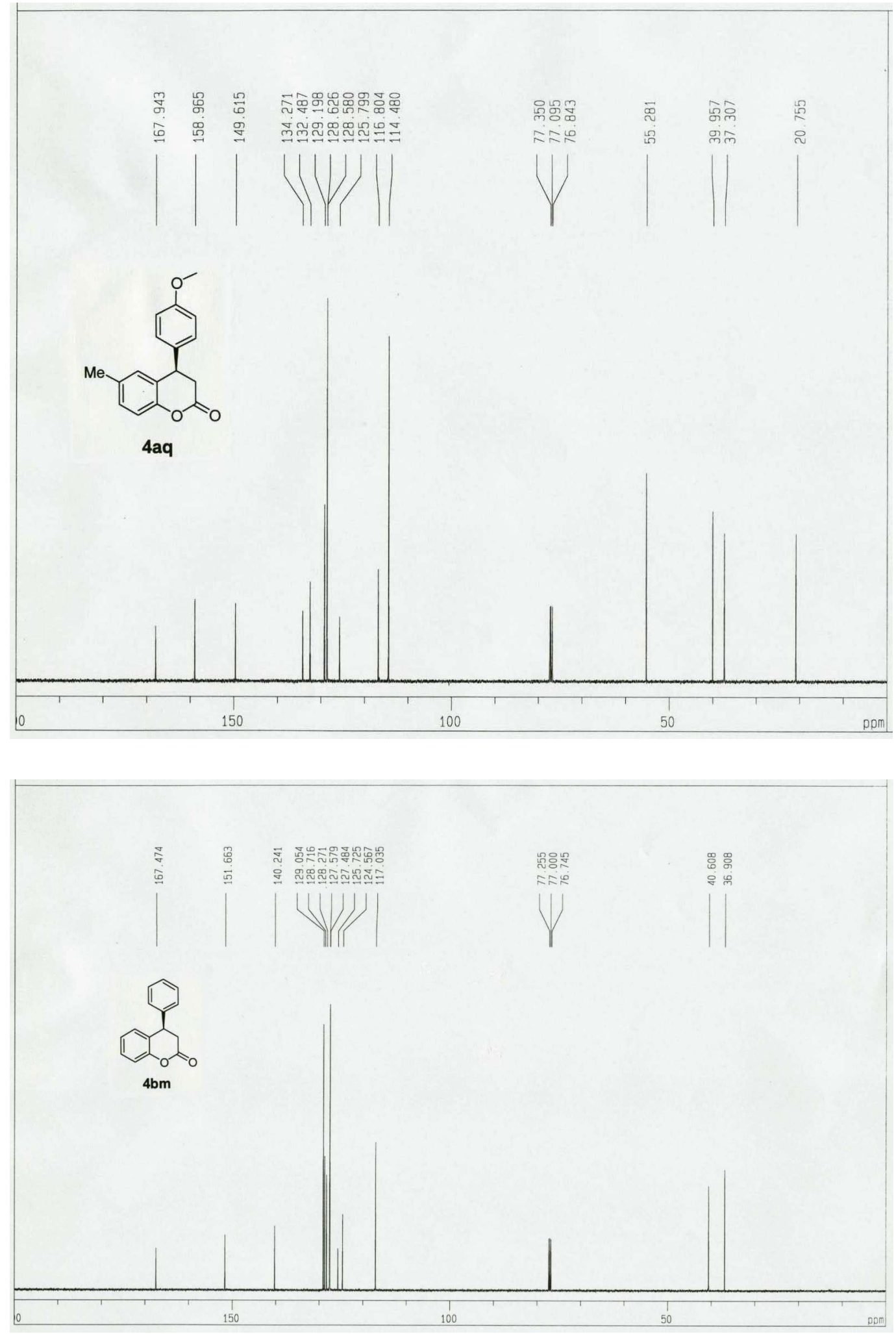

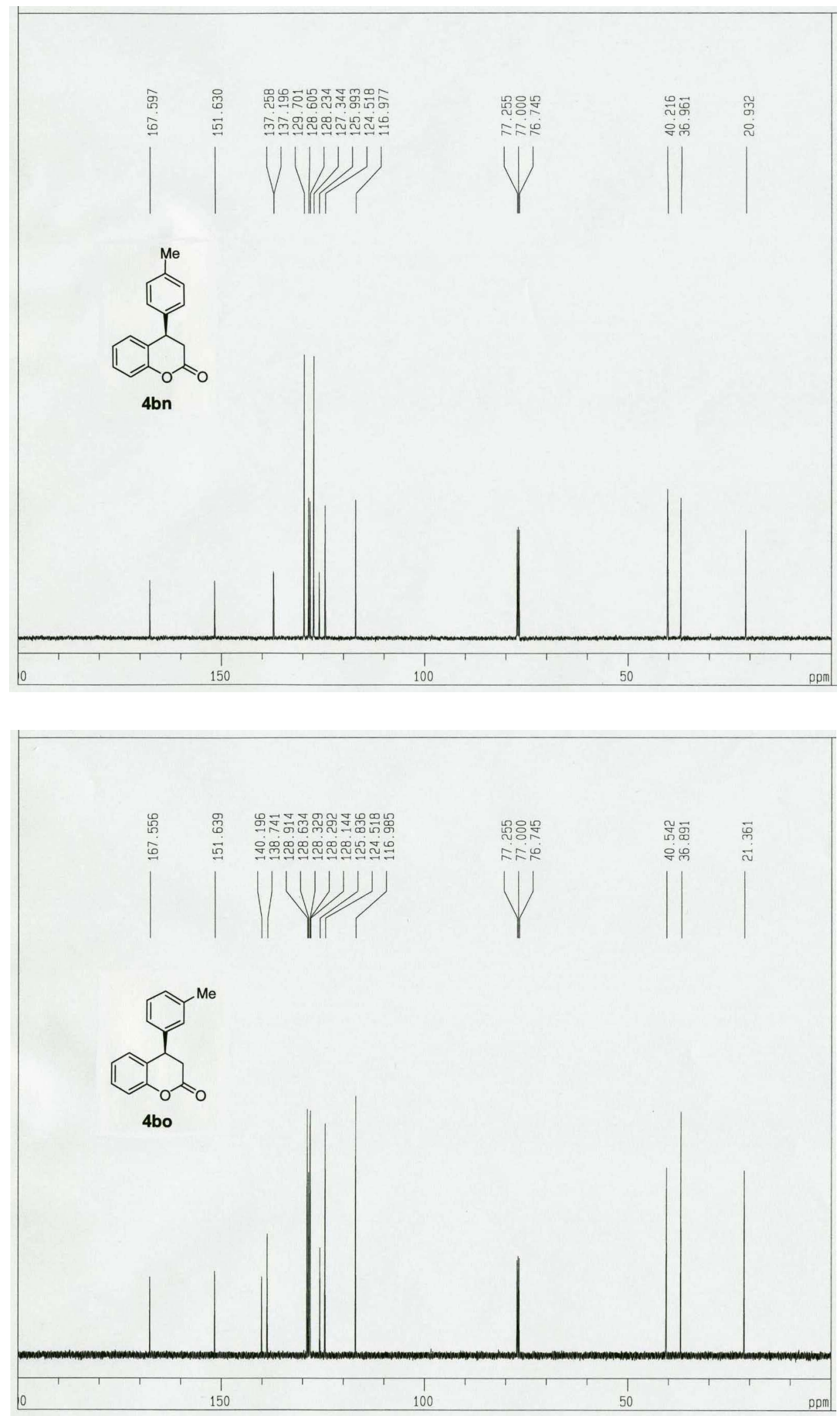

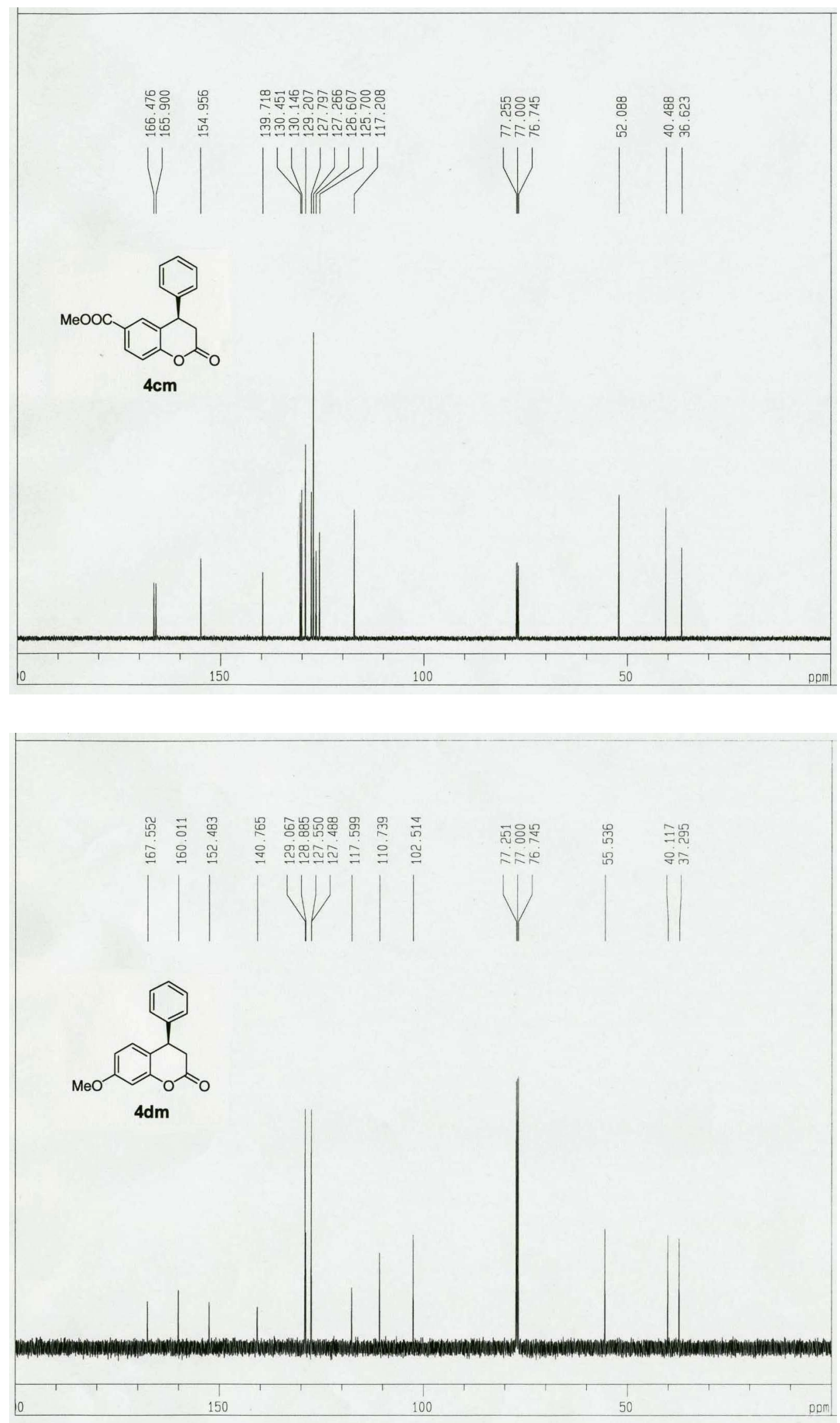

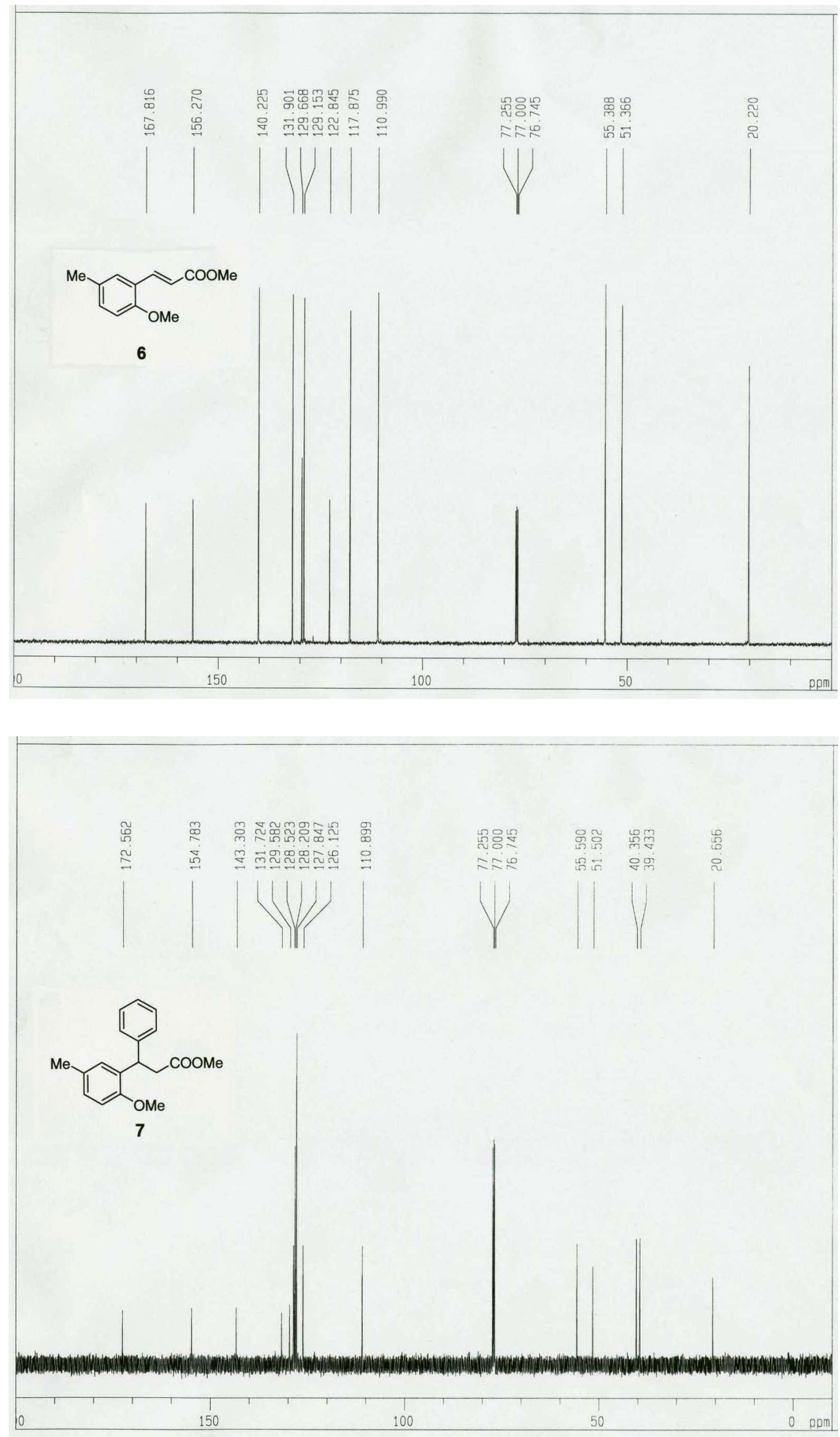


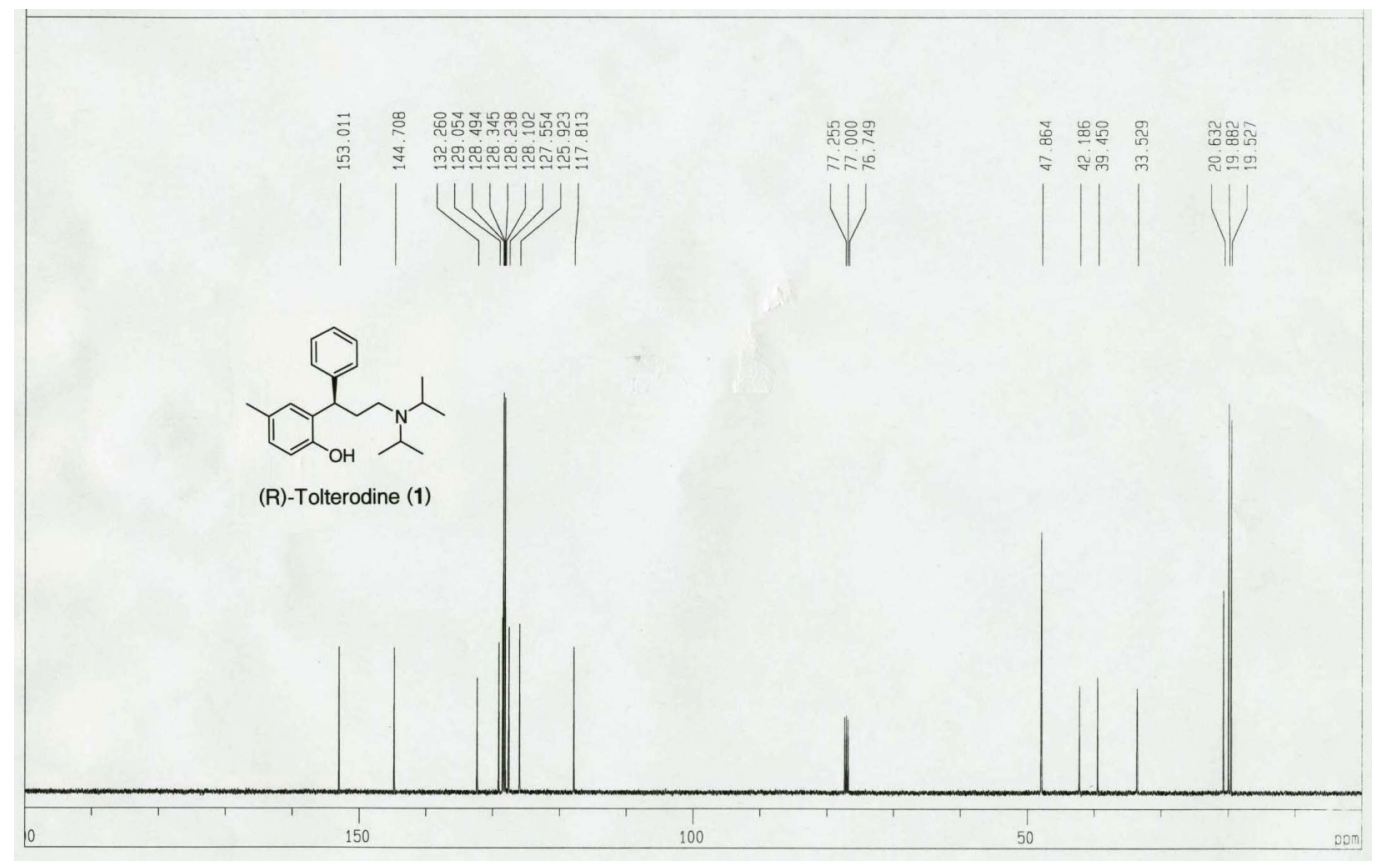

